\title{
Optimization of poly(vinyl chloride) concentration in aqueous solution and properties of dried film
}

\author{
Methakarn Jarnthong ${ }^{*}$, Lusheng Liao ${ }^{b}$, Fuquan Zhang ${ }^{c}$, Yueqiong Wang ${ }^{d}$, \\ Puwang $\mathrm{Li}^{\mathrm{e}}$, and Zheng Peng ${ }^{*}$
}

Key Laboratory of Tropical Crop Products Processing of Ministry of Agriculture, Agriculture Products

Processing Research Institute, Chinese Academy of

Tropical Agricultural Sciences, Zhanjiang 524001, China

amethakarn.jarnthong@yahoo.com, 'Isliao@qq.com, ’xqcyl2106@163.com, 'dyqw215@163.com,

epuwangli@163.com, 'zpengcatas@126.com

Keywords: Poly(vinyl alcohol), viscosity, processing, crystallization behavior.

Abstract. Rheological properties of poly(vinyl alcohol) (PVA) at various concentrations in aqueous medium were studied. The optimal concentration of PVA to obtain high solid content with low viscosity was determined. Films with different concentrations of PVA in aqueous solution have also been prepared. The influence of PVA concentration in solution on mechanical properties and thermal behavior of PVA films was investigated.

\section{Introduction}

Poly(vinyl alcohol) (PVA) is a water-soluble and biodegradable synthetic polymer with excellent film-forming, emulsifying, and adhesive properties, which widely used in different applications including electronics, biomedical, packaging, textile and food products [1-5].

Blending of PVA with other water-soluble polymers or latexes is interesting and easy way to improve their biodegradable property [6]. As a large number of solution and latex products are used in the form of films, high viscosities are undesirable from an applications point of view, while low solid content can lead to low drying rate which requires long time process. In synthetic latex research field, high solids content latexes with low viscosity have been studied by blending of mono-sized latexes [7-9]. It was showed that the lowest viscosity of $>65 \%$ solid content was obtained when the ratio of large to medium to small particles was approximately 80/10/10 (by weight) [7]. There have been very few studies examining rheological characterizations of polymer solution at different concentrations $[10,11]$. None of these have involved detailed the effect of concentration of polymer solution on the final properties of dried films.

It is important to understand the optimal concentration of PVA solution before blending with other polymers or fillers in order to provide high drying rate, low energy cost and improved mechanical and rheological properties of the final products. In this work, the influence of PVA concentration in water medium on rheological responses of PVA solutions was studied to determine the optimal concentration of PVA solution at high solid content with lowest possible viscosity, as well as mechanical properties and thermal behavior of the PVA films and discussed.

\section{Experimental}

Poly(vinyl alcohol) (PVA) (Sigma- Aldrich Inc., Saint Louis, USA) with average molecular weight of 85,000-124,000 and degree of hydrolysis varying from 87-89\% was used for the experiment without future purification. PVA solutions were prepared by mixing PVA and distilled water in relevant proportions $(1 \%, 3 \%, 5 \%, 7 \%$ and $10 \%(\mathrm{w} / \mathrm{w}))$. The mixture was stirred at $80^{\circ} \mathrm{C}$ to get clear homogeneous solutions. The PVA solutions were cast in Teflon mold and dried in vacuum oven at $30^{\circ} \mathrm{C}$ for 5 days.

Viscosity measurement was performed on a Brookfield viscometer (Brookfield, UK) with spindle 61 (speed 1.26, 3.14 and $6.28 \mathrm{rad} / \mathrm{s}$ ) at a constant temperature of $25^{\circ} \mathrm{C}$. The mechanical properties of all film samples were analyzed by a Hounsfield H10KS universal testing machine 
according to ASTM D412 at a crosshead speed of $500 \mathrm{~mm} / \mathrm{min}$ using five dumbbell specimens to obtain a medium value. Thermal properties of PVA films were measured by a DSC Q2000 differential calorimeter (TA Instruments) using heating rate $10^{\circ} \mathrm{C} \mathrm{min}^{-1}$ and temperature range from -85 to $100^{\circ} \mathrm{C}$ in nitrogen atmosphere. Percentage of crystallinity $\left(X_{c}\right)$ of PVA films was calculated from melting enthalpy of second heating run by the following equation:

$$
X_{c}(\%)=\frac{\Delta H_{f}}{\Delta H_{o}}
$$

where $\Delta H_{f}$ is the melting enthalpy measured in the heating experiments, $\Delta H_{0}$ is the theoretical value of enthalpy of $100 \%$ crystalline PVA which was taken as $138.6 \mathrm{~J} / \mathrm{g}$ [12].

\section{Results and discussion}

Viscosity measurement

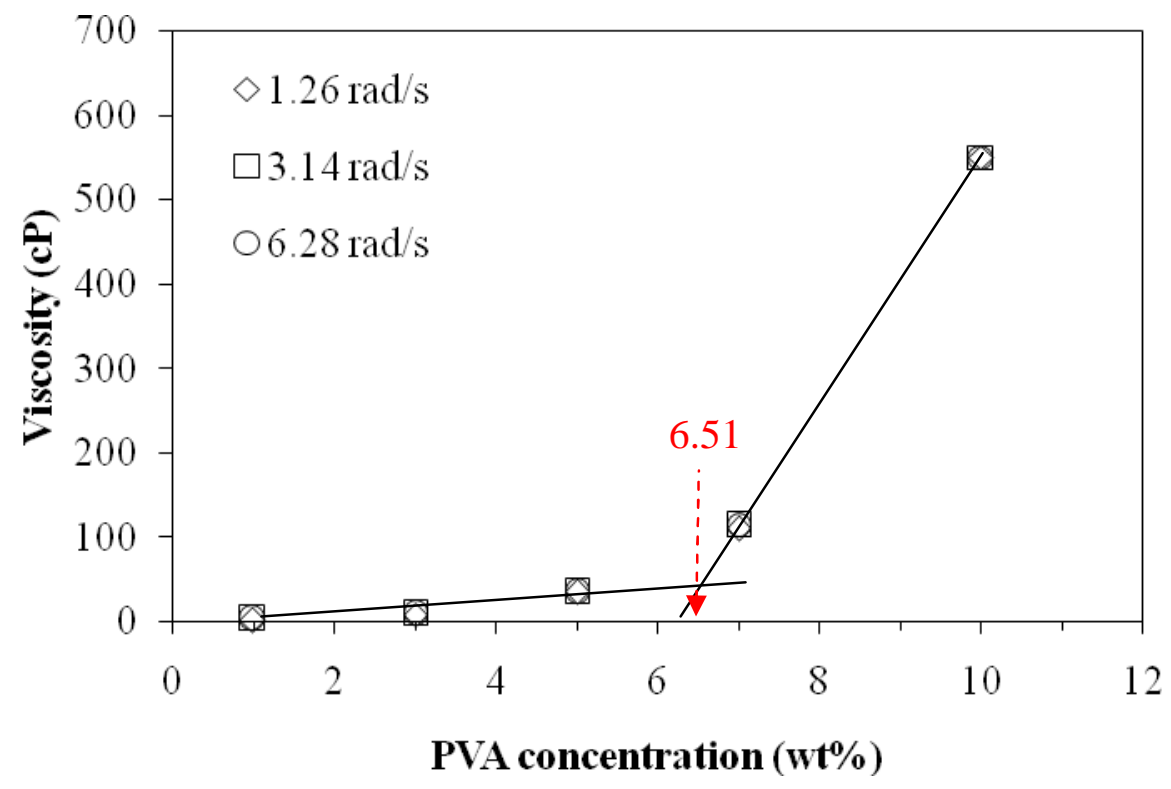

Fig. 1 Viscosity curve of PVA solutions at different concentrations.

Fig. 1, the increase of viscosity of PVA solution with increasing concentration is observed. It is seen that the viscosity of PVA solution starts to increase at $7 \mathrm{wt} \%$ and tends toward infinity when PVA concentration reach $10 \mathrm{wt} \%$. From viscosity curve, the intercept of 2 linear lines with different slopes for low and high viscosities was at $6.51 \mathrm{wt} \%$ and viscosity of $43.7 \mathrm{cP}$, indicating the highest solid content of PVA in water solution at the lowest possible viscosity.

\section{Thermal analysis}

The thermal characteristics including the crystallization temperature $\left(T_{c}\right)$, glass transition temperature $\left(T_{g}\right)$, melting temperature $\left(T_{m}\right)$, melting enthalpy $\left(\Delta H_{f}\right)$, and degree of crystallization $\left(X_{c}\right)$ of the commercial PVA and the PVA films prepared from different concentrations are summarized in Table 1. 
Table 1 The thermal characteristics of the commercial PVA and the PVA films.

\begin{tabular}{|l|c|c|c|c|c|}
\hline \multicolumn{1}{|c|}{ Samples } & $T_{c}$ & $T_{g}$ & $T_{m}$ & $\Delta H_{f}$ & $X_{c}$ \\
\hline Commercial PVA & 110.8 & 72.6 & 166.2 & 25.0 & 18.02 \\
\hline $1 \mathrm{wt} \%$ & 118.4 & 74.3 & 172.2 & 24.7 & 17.79 \\
\hline $3 \mathrm{wt} \%$ & 121.3 & 74.4 & 173.5 & 25.3 & 18.25 \\
\hline $5 \mathrm{wt} \%$ & 127.1 & 74.5 & 175.2 & 27.0 & 19.48 \\
\hline $7 \mathrm{wt} \%$ & 133.6 & 74.5 & 181.7 & 29.1 & 21.00 \\
\hline $10 \mathrm{wt} \%$ & 147.4 & 74.4 & 186.9 & 33.7 & 24.31 \\
\hline
\end{tabular}

As shown in Table 1, significant changes can be observed in the $T_{c}, T_{g}, T_{m}$, and $X_{c}$ of PVA with different concentrations of PVA solution. The shift in $T_{c}$ and $T_{m}$ toward higher temperatures of the PVA films indicates the increase of crystallinity due to the plasticising effect of the water [13].

Fig. 2 shows the DSC cooling (A) and heating (B) thermograms for commercial PVA and the PVA films. Commercial PVA exhibited clear $T_{g}$ at $72.6^{\circ} \mathrm{C}$ and prominent melting endotherm at around $166.2^{\circ} \mathrm{C}$, indicating a semicrystalline structure. The influence of molecular level interactions between PVA and water were reported to mostly relate with amorphous region in PVA $[14,15]$. The water molecules diffuse initially through the amorphous region of the PVA and attach themselves to hydroxyl side groups, disrupting inter and intra molecular hydrogen bonding, and swelling the PVA. Finally, the free water existing in the PVA molecules leads to the reduction of crystalline region.
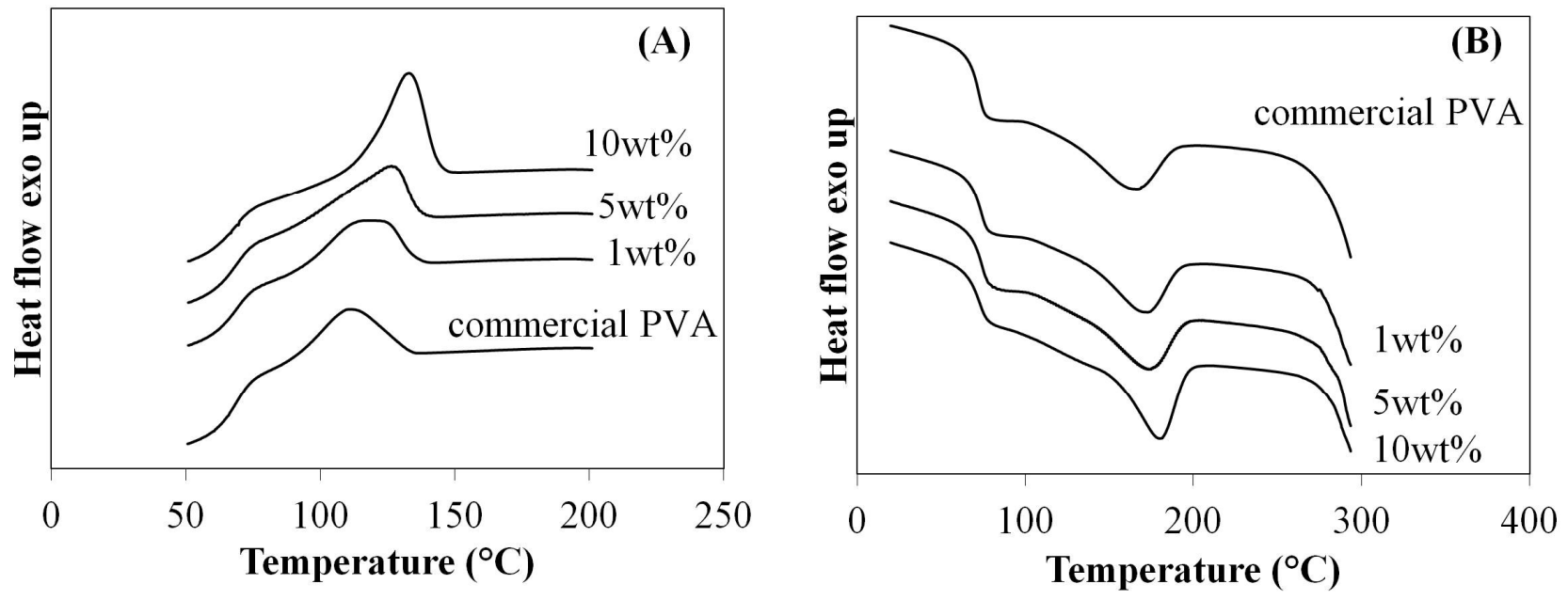

Fig. 2 DSC thermogram during (A) cooling and (B) heating of commercial PVA and PVA films.

At low concentration of PVA, free water, which is presumably located in free volume within the PVA, induces hydrogen bonding between water and PVA molecules and destroys crystallinity by forcing the chains to change configuration from the crystalline planar zig-zag into an amorphous configuration in order to accommodate the effective increase in size of the side group, and because of the destruction of hydrogen bonding in the system which acts to hold the chains together in a tight, crystalline array [15]. Therefore, the PVA film prepared by lower concentration of PVA might contain more amount of free water resulting in diminution of crystallinity of dried film.

\section{Mechanical properties}

As seen in Fig. 3, the tensile strength of the PVA films was significantly increased with increasing PVA concentration in solution, while the elongation was decreased. Since PVA is a semicrystalline polymer, its mechanical properties strongly depend on the degree of its crystallinity. 


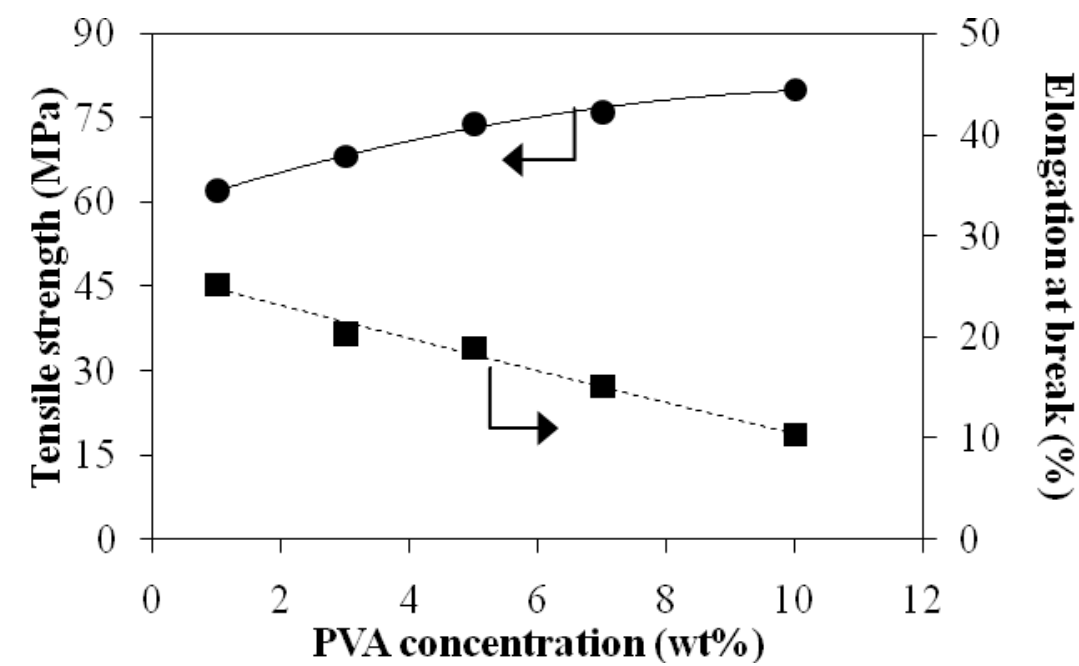

Fig. 3 Mechanical properties of PVA films at different concentrations.

\section{Conclusions}

The highest solid content of PVA (MW 85,000-124,000) in water solution with the lowest viscosity was observed at $6.51 \mathrm{wt} \%$ PVA (viscosity of $43.7 \mathrm{cP}$ ). The thermal analysis showed that the crystallinity of PVA increased with increasing concentration of PVA in the solution, which leading to enhanced tensile strength of PVA films.

\section{Acknowledgements}

This research was supported by the National Natural Science Foundation of China (51603230); Modern Agricultural Talent Support Program; Natural Science Foundation of Hainan Province (2017CXTD017); Natural Science Foundation of Hainan Province (2017CXTD017); Central Public-interest Scientific Institution Basal Research Fund for Chinese Academy of Tropical Agricultural Sciences (1630122017T12).

\section{References}

[1] R. Gangopadhyay, A. De, G. Ghosh: Synthetic Metals 123(2001), p. 21-31

[2] J. Jobish, N. Charoen, P. Praveen: Journal of Non-Crystalline Solids 358(2012), p. 1113-1119

[3] O. Probst, E. M. Moore, D. E. Resasco, B. P. Grady: Polymer 45(2004), p. 4437-4443

[4] K. Strawhecker, E. Manias: Chemistry of materials 12(2000), p. 2943-2949

[5] X. Zhao, Q. Zhang, D. Chen, P. Lu: Macromolecules 43(2010), p. 2357-2363

[6] Z. Guohua, L. Ya, F. Cuilan, Z. Min, Z. Caiqiong, C. Zongdao: Polymer Degradation and stability 91(2006), p. 703-711

[7] F. Chu, A. Guyot: Colloid \& Polymer Science 279(2001), p. 361-367

[8] K. Ouzineb, C. Graillat, T. McKenna: Journal of applied polymer science 97(2005), p. 745-752

[9] M. Schneider, J. Claverie, C. Graillat, T. McKenna: Journal of Applied Polymer Science 84(2002), p. 1878-1896

[10]S. K. Mallapragada, N. A. Peppas: Journal of Polymer Science-B-Polymer Physics Edition 34(1996), p. 1339-1346

[11] M. Mucha: Reactive and Functional Polymers 38(1998), p. 19-25

[12]S. El-Sayed, K. Mahmoud, A. Fatah, A. Hassen: Physica B: Condensed Matter 406(2011), p. 4068-4076

[13]H. E. Assender, A. H. Windle: Polymer 39(1998), p. 4295-4302

[14]R. Hodge, T. Bastow, G. Edward, G. Simon, A. Hill: Macromolecules 29(1996), p. 8137-8143

[15]R. Hodge, G. H. Edward, G. P. Simon: Polymer 37(1996), p. 1371-1376 